\title{
May-Thurner syndrome
}

\author{
Santhosh Poyyamoli ${ }^{1}$, Pankaj Mehta ${ }^{2}$, Mathew Cherian ${ }^{1}$, Rinoy R. Anand ${ }^{1}$, Santosh B. Patil ${ }^{1}$, \\ Sanjeeva Kalva ${ }^{3}$, Gloria Salazar ${ }^{3}$
}

${ }^{1}$ Department of Diagnostic and Interventional Radiology, Kovai Medical Center and Hospital, Coimbatore, Tamil Nadu, India; ${ }^{2}$ Department of Radiology, KMCH Institute of Health Sciences and Research, Coimbatore, Tamil Nadu, India; ${ }^{3}$ Division of Interventional Radiology, Massachusetts General Hospital Boston, Harvard University, MA, USA

Contributions: (I) Conception and design: P Mehta, G Salazar; (II) Administrative support: M Cherian, P Mehta, G Salazar; (III) Provision of study materials or patients: None; (IV) Collection and assembly of data: S Poyyamoli, SB Patil, RR Anand; (V) Data analysis and interpretation: None; (VI) Manuscript writing: All authors; (VII) Final approval of manuscript: All authors.

Correspondence to: Pankaj Mehta. KMCH Institute of Health Sciences and Research, Coimbatore, Tamil Nadu, India.

Email: drpankajmehta@kmchhospitals.com.

\begin{abstract}
May-Thurner syndrome (MTS) is a venous compression syndrome in which the left common iliac vein (LCIV) is compressed between the lower lumbar spine and the right common iliac artery (RCIA). Variations are known where in the right lower limb can be affected. While most of the cases are asymptomatic, it can cause severe morbidity in symptomatic individuals, most commonly deep vein thrombosis and post thrombotic sequelae. In this article, we review the key clinical features, multimodality imaging findings and treatment options of this disorder. Our goal is to raise awareness of this underdiagnosed condition among clinicians in order to promote early detection and recognition to enhance positive and expedited outcomes.
\end{abstract}

Keywords: Compressive venous syndromes; Cockett syndrome; May-Thurner syndrome (MTS)

Submitted Jan 14, 2020. Accepted for publication Mar 09, 2020.

doi: $10.21037 / \mathrm{cdt} .2020 .03 .07$

View this article at: http://dx.doi.org/10.21037/cdt.2020.03.07

\section{Introduction}

May-Thurner syndrome (MTS), also known as ilio-caval compression syndrome or iliac vein compression syndrome or Cockett syndrome is a clinical condition resulting from compression of the ilio-caval anatomical unit against the underlying bone by the overlying arterial system. The most common lesion is the compression of the left common iliac vein (LCIV) between the overlying right common iliac artery (RCIA) and the fifth lumbar vertebra. Other forms of compression also exist, such as the right sided MTS and caval compression $(1,2)$. Most commonly presenting in adult women in their $3^{\text {rd }}$ to $5^{\text {th }}$ decade, the clinical presentation of MTS is variable and diverse. The management revolves around improving venous outflow from the affected limb. Minimally invasive endovascular procedures form the mainstay of treatment.

\section{Pathophysiology}

The occurrence of iliac vein compression is known from a long time. As early as 1851 , Virchow noted that the compression of the LCIV by the RCIA was the cause of increased incidence of deep venous thrombosis (DVT) in the left lower limb (3). McMurrich, in his study of autopsied patients in 1908 noted that left sided iliac veins were more commonly obstructed than the right and deemed it to be congenital (4). Many years later, in a large study published by Ehrich and Krumbhaar, the congenital nature of this lesion was challenged, and they attributed the obstruction to an acquired venous valve defect (5).

The landmark study by May and Thurner in 1957 attributed this pathology to a thickening of the iliac vein wall by continuous irritation by pulsation of the overlying artery and they coined the term "spur" (6). In 1965, 
Cockett and Thomas studied 29 patients for proximal vein thrombosis by per-trochanteric venography augmented by direct femoral vein venography and observed the stenosis/ flattening of LCIV. They also confirmed surgically the venous strictures and found that simple decompression does not re-canalize the vein. They also found that patch venoplasty was the best treatment for these patients (7).

The classical lesion is an extrinsic compression of the LCIV by the RCIA against the lumbar vertebra. Many authors address to this as May Thurner anatomy and do not associate it with any hemodynamic component (8). Though many people may harbor this anatomy, only a few people become symptomatic.

The term MTS is reserved for the symptomatic MT anatomy with symptoms of chronic venous insufficiency and a venous stenosis or obstruction accompanied by collaterals with or without DVT. The RCIA is considered to cause this partial obstruction by direct mechanical compression of the LCIV and also, the intimal hyperplasia in the LCIV due to the shear stress on the anterior and posterior walls of the LCIV secondary to the pulsation of the overlying RCIA contributes to venous obstruction and subsequent thrombosis (9).

\section{Clinical presentation}

MTS commonly affects women in their third to fifth decades of life, though is not confined to this group (6). The commonest presentation of these patients is that of venous hypertension. Patients may present with acute episodic (activity related) heaviness and swelling of left lower limb or venous claudication which is relieved with rest and leg elevation. This progresses to chronic venous insufficiency presenting with features of varicose veins, leg pigmentation or venous ulcers. A high degree of suspicion must be maintained to investigate these patients for iliac vein compression $(10,11)$.

Many a times, patients are investigated for iliac vein compression after standard ablative treatment for varicose veins such as saphenous vein ablation or perforator ablation fails to give the satisfactory results.

Sometimes, the first presentation may be that of DVT with painful left lower limb swelling. There may be no precipitating cause or the disease may first present during or after pregnancy $(12,13)$. A history of recent use of oral contraceptive pills may be elicited (14).

\section{Diagnosis}

Unilateral swelling of the lower limb is commonly interrogated by physical examination aided by a venous duplex ultrasound of the affected limb. While color Doppler ultrasound is adequate and sensitive for the diagnosis of deep vein thrombosis and evaluation of varicose veins and venous incompetence, it is woefully inadequate to study the pelvic veins, especially the ilio-caval region. If the ultrasound is able to visualize the proximal CIV, it may demonstrate the lack or reduction of phasic variations of blood flow distal to the stenosis and turbulence (color mosaic) at the site of stenosis (Figure 1). The opposite side serves as a control. Venous collaterals, an important component of the diagnostic findings are poorly identified by ultrasound.

If MTS is suspected, given the right clinical setting, cross sectional imaging is necessary to evaluate the iliocaval region to identify the stenotic lesion. Multi-detector computed tomography (MDCT) venography or magnetic resonance $(M R)$ venography are excellent imaging modalities to detect MT anatomy and demonstrate the presence of collaterals. Both, CT and MR venography have greater than 95 percent sensitivity and specificity for diagnosing MTS (15-17). Both the modalities have the added benefit of detecting spinal degeneration and anomalies of bone and vascular structures and other pelvic pathologies.

MDCT venography demonstrates the compression of the LCIV by the RCIA, presence of bony spurs, deep vein thrombosis, and pelvic venous collaterals and helps in the exclusion of other compressive pathologies (Figure 2). Patients with suspicion of pulmonary embolism can also undergo a CT pulmonary angiogram in the same sitting. Jeon et al. (18) proposed a morphological classification based on CT venography which included 3 types-type 1 was focal compression of the iliac vein by the artery, type 2 was diffuse atrophy of the iliac vein up to its formation, and type 3 was a thin, cord-like iliac vein in its entire length. This classification has a bearing on the treatment strategy as the fibrotic ones pose a challenge for balloon dilatation prior to stent placement.

MR venography, though uncommonly done for diagnosing MTS, is as accurate as CT in diagnosing the vascular compression, deep vein thrombosis and pelvic venous collaterals. In addition to the post contrast $M R$ 

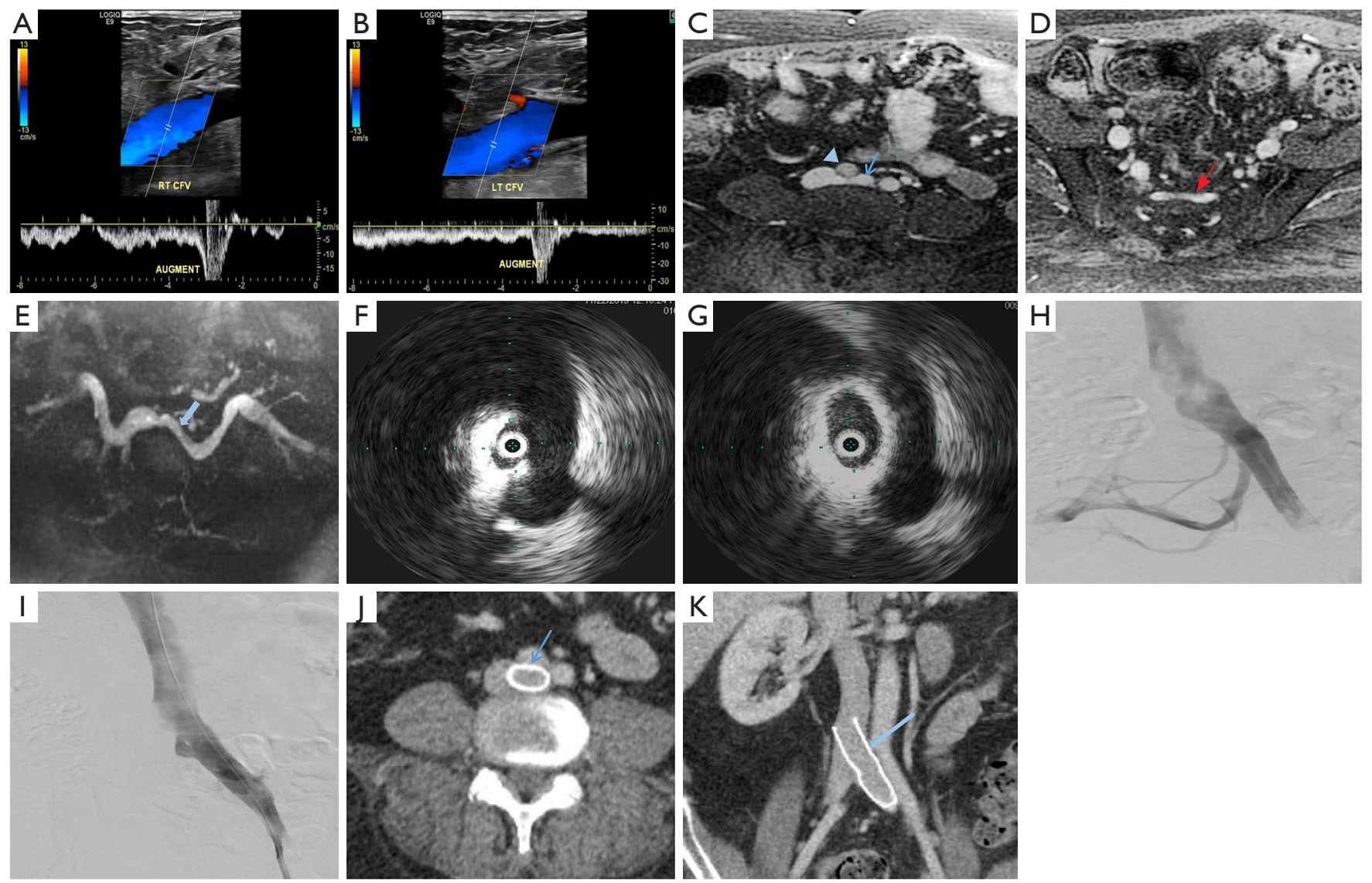

Figure 1 A 51-year-old male with left leg swelling and prior history of recurrent left leg DVT. USG color Doppler showed normal respiratory variation in right common femoral vein (A) and diminished respiratory variation in left common femoral vein suggesting proximal obstructive lesion (B). Post contrast MR venogram (C,D) demonstrated compression of left common iliac vein (C, arrow) by right common iliac artery (C, arrowhead) and cross pelvic collaterals (arrow) (D). Axial MIP time-of-flight MR image (E) showed hemodynamically significant left common iliac vein compression (arrow) with non-visualized left internal iliac vein suggesting flow reversal in left internal iliac vein. Intravascular ultrasound (F) showed small caliber left common iliac vein at the site of crossing by right common iliac artery. Post stenting intravascular ultrasound $(\mathrm{G})$ showed restoration of lumen of the left common iliac vein. Catheter venogram $(\mathrm{H})$ showed significant narrowing of left common iliac vein with cross pelvic collaterals. Post deployment of VICI (Boston Scientific, USA) stent (I), there was restoration of lumen with absence of pelvic collaterals. Three months after stenting, axial (J) and coronal (K) reformatted images of MDCT venography showed widely patent stent (arrow) with normal lumen of the left common iliac vein. The patient's symptoms of left leg swelling resolved at 3 months. DVT, deep venous thrombosis; USG, ultrasonography; MR, magnetic resonance; MIP, maximum intensity projection.

venography study (Figure 1), a time-of-flight (TOF) sequence (Figure 1) may identify flow reversal in the internal iliac vein (as such, no signal on $2 \mathrm{D}$ TOF) ipsilateral to common iliac vein compression and enlarged ascending lumbar vein in case of a tight iliac stenosis (19). Newer techniques like 4D flow MR venography may provide interesting insights into the flow dynamics like velocity and direction with superior temporal resolution in these patients; however there is lack of comprehensive evidence
(20,21). One disadvantage of extracellular MR contrast agents is that they behave like iodinated contrast media with limited time window for imaging and need for repeat contrast administration if the initial imaging is suboptimal. This apparent glitch can be overcome by using blood-pool contrast agents (BPCA) which can remain in circulation for prolonged periods and thus allow a reliable and repeatable examination (22). Gadofosveset trisodium (Ablavar ${ }^{\circledR}$, Lantheus Medical Imaging, North Billerica, MA) is the 

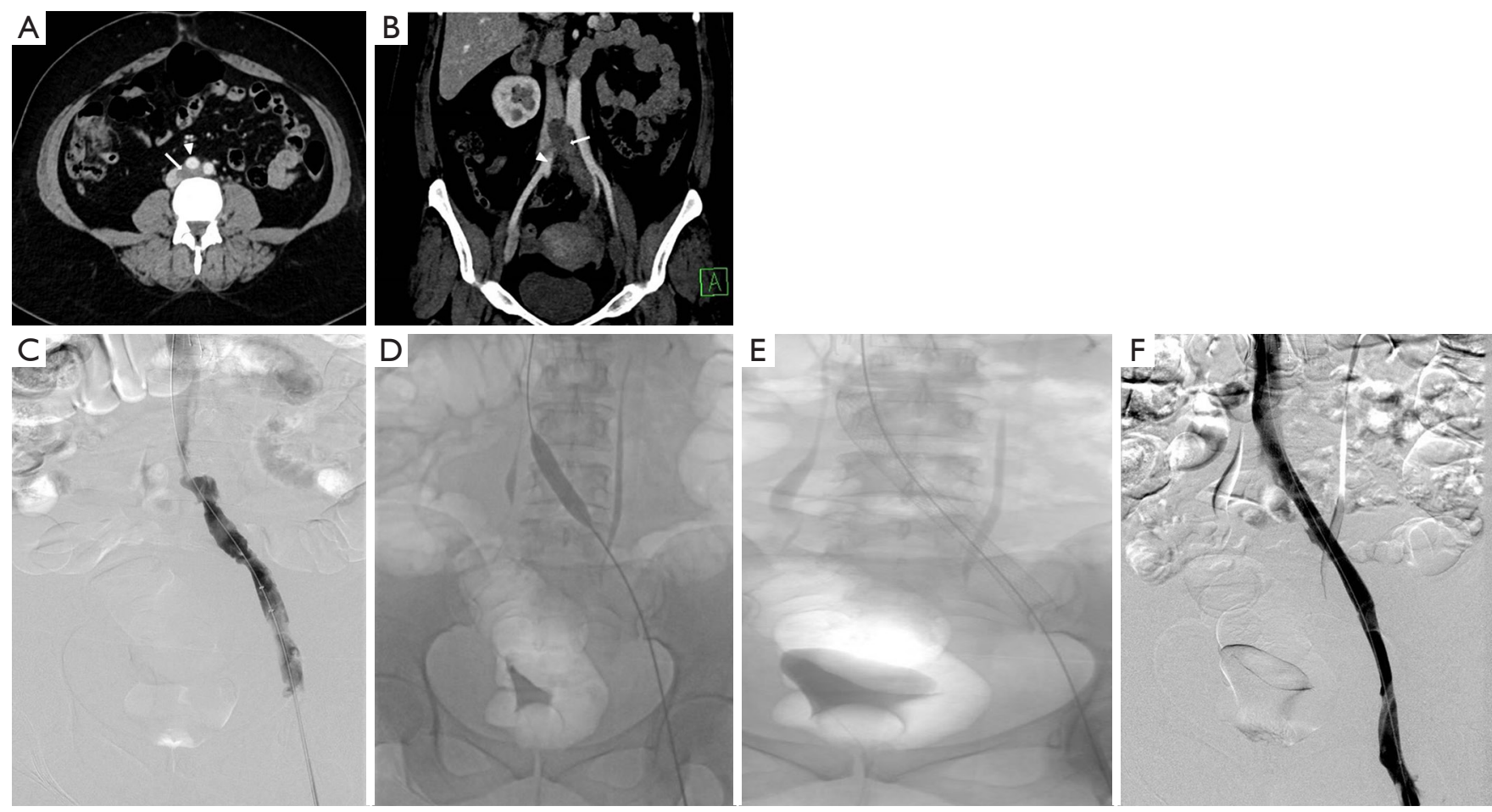

Figure 2 Axial (A) and coronal (B) reformatted images of MDCT venography of a 37 years old female with left lower limb swelling showing compression of left common iliac vein (arrow) by right common iliac artery (arrowhead) with thrombus in left common iliac vein. Suction thrombectomy was performed using Angiojet (Boston Scientific, USA). Catheter venogram (C) performed after suction thrombectomy showed proximal left common iliac vein stenosis. Balloon dilatation (D) was performed using 8mm balloon. Self- expanding stainless steel stent (Wallstent, Boston Scientific, USA) (E,F) was deployed with restoration of caliber and flow across left common iliac vein. MDCT, multi-detector computed tomography.

most commonly used BPCA for venous imaging. Another agent that has been used as a blood-pool contrast offlabel is Ferumoxytol, a superparamagnetic intravenous iron supplement, FDA approved for patients with anemia of renal failure. The contrast effect is due to the strong shortening of $\mathrm{T} 1$ and $\mathrm{T} 2{ }^{*}$ by the iron supplement. Circulation times are longer owing to its larger size and carbohydrate coating, making it suitable as a blood pool agent.

The finding of iliac vein compression in a solitary CT/ MR examination should be interpreted with caution and corroborated with a prone scan if feasible, as the volume status of the individual influences the degree of compression of the iliac vein. Hence, it is recommended that the patient is well hydrated to achieve optimal volume status before any cross sectional imaging (23). Hydrating the patient with 1-2 litres orally within one hour prior to imaging has been shown to improve the specificity of diagnosis (24). It is estimated that iliac vein compression of $65 \%$ or less may be seen in normal individuals (25). As such, demonstration of pelvic venous collaterals and identification of iliac vein spur are important to assess the hemodynamic significance of iliac vein compression.

In the past decade, intra-vascular ultrasound (IVUS) has steadily found increasing use in the diagnosis and management of MTS. During the interventional procedure, IVUS can help in documenting and localizing the cause of iliac venous obstruction-spurs and intimal lesions from chronic adherent thrombus. It can also show the dynamic compression by the overlying artery (Figure 1). In addition to helping in re-canalizing the chronic occlusion, it can assess response of MT lesion to angioplasty and stent placement (Figure 1) and presence of residual thrombus thus aiding in continuation of lysis.

Catheter venography has traditionally been considered the gold standard for diagnosis of MTS. However due to its invasive nature, it is nowadays reserved for cases in which endovascular intervention is planned or diagnosis 
by non-invasive modalities is equivocal. Venography can demonstrate acute thrombosis within the iliac vein or the ilio-femoral veins. Chronic cases are associated with stenosis or occlusion of LCIV with pelvic and retroperitoneal collaterals (Figure 1) and flow reversal in ipsilateral internal iliac vein. Hemodynamic pressure gradient measurement is a valuable adjunct in confirming presence of significant stenotic lesion associated with MTS. May and Thurner (6) advocated measurement of pressure difference between the iliac veins ( $2 \mathrm{mmHg}$ at rest, $3 \mathrm{mmHg}$ during exercise), whereas Ferris et al. (26) advocated any gradient between the IVC and left iliac vein for diagnosis of hemodynamically significant obstruction.

\section{Management}

Patients with May-Thurner anatomy largely remain asymptomatic or do not seek medical advice for mild or non-troubling symptoms. In the minority of patients with symptoms, who are branded as MTS, there are two patient groups. The first group manifests with acute or recurrent proximal DVT, commonly involving the left lower limb, often unprovoked or with trivial triggers. The second group has a spectrum of clinical presentations of chronic venous insufficiency.

Among patients presenting with acute proximal DVT ( $<14$ days duration), MTS should be suspected if there are risk factors such as young age, female sex, oral contraceptive intake, left lower limb involvement, etc. Careful evaluation with non-invasive imaging (Doppler, CT or MR venography) should be performed prior to consideration of endovascular therapy. Secondary causes such as pelvic mass or pregnancy have to be looked for, apart from assessing the extent of DVT.

The results of ATTRACT trial (27) suggested that catheter directed thrombolysis was not superior to systemic anticoagulation in patients with acute lower limb DVT. However, in the subgroup of DVT patients with MTS, clearing the thrombus load and establishing an outflow still forms the cornerstone of management (24-26). Patients who are eligible for thrombolysis should be strongly considered for pharmaco-mechanical thrombolysis for maximal thrombus clearance and assessment of underlying iliac stenosis, followed by angioplasty and stenting of the underlying stenotic lesion. Isolated catheter directed thrombolysis or mechanical thrombectomy can be chosen based on individual patient factors, expertise and availability of the devices. Newer techniques such as rheolytic thrombectomy with Angiojet (Boston Scientific, USA) or rotational thrombectomy (Cleaner-XT, Argon medical, USA) are additional options.

All patients of MTS with acute DVT should be started on anticoagulation at the first instance. The choice of initial anticoagulation is an intravenous infusion of unfractionated heparin titrated against activated partial thromboplastin time (aPTT). The use of a prophylactic IVC filter before a definitive clot removal procedure is not recommended (28). Some authors suggest use of IVC filters in the presence of large volume or free floating IVC thrombus, resistance or contraindication for anticoagulation or history of recurrent pulmonary embolism. If needed, an IVC filter is placed through the contralateral femoral access or jugular venous access. However, the incidence of pulmonary embolism is very low in MTS due to the protective effect of venous stenosis (29).

Patients with isolated iliac vein thrombosis (minority) can have an ipsilateral common femoral venous access for the procedure. More commonly though, the femoral and popliteal veins are simultaneously involved along with the iliac vein, requiring a popliteal venous access. The choice of sheath size depends on the technique of recanalization. Various catheters (e.g., Indigo, Penumbra, USA) and lytic agents (Alteplase or Urokinase) can be used based on operator preference. During catheter directed thrombolysis, the sheath is flushed with heparin as a continuous infusion. Once there is near total or reasonable recanalization, the underlying stenosis at the iliac venous segment can be unmasked (Figure 2). Caution should be exercised in grading venous stenosis during venography as the iliac vein is compressed or flattened anteroposteriorly leading to a "pancake" effect on the angiographic image in the frontal projection (29). Various oblique projections may be needed for accurate estimation of stenosis. Intravascular ultrasound (IVUS) can help in locating and grading the stenosis. An appropriately long balloon of $8 \mathrm{~mm}$ or $10 \mathrm{~mm}$ diameter is used to perform an initial angioplasty (Figure 2). The results of angioplasty alone are traditionally below par when compared with stenting, irrespective of the post angioplasty result (30). At this point, an IVUS can help assess the response to plain balloon angioplasty and confirm the landing zones for the stent if required. Self-expanding, Stainless steel stents (Wallstent, Boston Scientific, USA) are preferred, because of their higher radial force, long length and flexibility (Figure 2). However, these stents come with niggles of their own; difficulty in precise placement and possibility of foreshortening being a few. As a principle, 
balloon mounted stents should be avoided as they can be deformed by the arterial pulsation. Multiple stents should also be avoided as they increase the incidence of in-stent stenosis (31). The primary patency rates at 1 year and 5 years were $93.2 \%$ and $74.5 \%$ respectively and the secondary patency rates at $1-$ and 5 -year at $100 \%$ and $92 \%$ respectively in one single center experience (31). The 6-year primary patency rate of iliac venous stents is close to $79 \%$, which exemplifies the role of stenting in this setting (32). The distal (downstream) landing zone is still a debatable topic (33). The Wallstent, being a braided stent, is weakest at its ends and this makes the stent vulnerable to being crushed by the culprit RCIA. Hence the interventionists overcome this by landing it in the lowermost vena cava apposing the right lateral wall. But the problem with this technique is it induces a pseudo-intima formation over the caged right common iliac vein surface of the stent resulting in increased incidence of venous thrombosis on the covered side. Newer dedicated laser-cut nitinol venous stents such as the VICI (Boston Scientific) (Figure 1) (34) and VENOVO (Bard) (35) stents have high patency rates and excellent precision at the time of deployment. These stents have higher resistive radial strength preventing the collapse or crushing of the stent by the right common iliac artery and hence can be deployed flush with the ostium of the LCIV. Following the procedure, patients are switched to oral anticoagulation for a period of 6 months and low dose aspirin thereafter. The choice of oral anticoagulation post endovenous therapy is not adequately evaluated in literature. A systematic review of the studies examining endovenous therapy for MTS, revealed that warfarin has been the most commonly used anticoagulant (36). The newer oral anticoagulants, apart from being equally efficacious, certainly have benefits over warfarin including reduced bleeding complications and avoidance of periodic monitoring. Periodic follow up with ultrasound or CT venography at $1,3,6$ and 12 months and thereafter yearly for stent patency and symptomatic recurrence is important for early diagnosis of stent occlusion and retreatment. Treatment failures are very rare unless there is residual stenosis anywhere in the dilated/stented segment, incomplete clot clearance, suboptimal inflow from femoropopliteal veins or noncompliance to optimal anticoagulation. An immediate treatment failure in the form of acute stent re-thrombosis is addressed with a relook endovenous therapy with correction of the causative factor. Delayed presentation with stent occlusion is approached according to the clinical findings. If the symptoms are subacute or chronic, of mild severity, usually after cessation of anticoagulation, with well collateralized stent occlusion, then a conservative strategy with reinitiation of lifelong anticoagulation is advised. Alternatively, if there is acute onset severely symptomatic venous thrombosis, a repeat endovenous intervention is preferred.

MTS patients presenting with chronic venous insufficiency (recurrent edema, dermal changes, and superficial venous reflux) will also benefit from angioplasty and stenting (26). These patients can undergo noninvasive imaging (MDCT/MR venography) to establish the diagnosis. Superficial venous reflux disease can be treated with ablative techniques with simultaneous treatment of the iliac venous stenosis (37). After the procedure, the patient can be put on dual anti-platelets for a period of 6 months and low dose aspirin indefinitely. All patients should be advised to wear thigh or knee-high compression stockings to prevent recurrence of symptoms.

Surgical treatment, although feasible, is not preferred, as it is associated with morbidity and poor results. Femoral venotomy and clot de-bulking can be done if there is a contraindication for thrombolysis, but it is seldom performed these days with advancements in endovascular thrombectomy techniques.

\section{Conclusions}

MTS is an under-diagnosed entity and should be considered when evaluating left lower limb DVT. A low threshold for cross sectional imaging such as MDCT venography and MR venography should be maintained in the correct clinical setting. Early diagnosis and appropriate treatment with pharmaco-mechanical thrombolysis with angioplasty and stenting will minimize the delayed morbidity of postthrombotic syndrome.

\section{Acknowledgments}

Funding: None.

\section{Footnote}

Provenance and Peer Review: This article was commissioned by the editorial office, Cardiovascular Diagnosis and Therapy for the series "Compressive Vascular Syndromes". The article has undergone external peer review.

Conflicts of Interest: All authors have completed the ICMJE 
uniform disclosure form (available at http://dx.doi. org/10.21037/cdt.2020.03.07). The series "Compressive Vascular Syndromes" was commissioned by the editorial office without any funding or sponsorship. Dr. SK served as the unpaid Guest Editor of the series. Dr. SK reports personal fees from Elsevier, personal fees from Springer, personal fees from Koo Foundation, Taiwan, personal fees from Medtronic Inc, personal fees from Penumbra Inc, personal fees from US Vascular, other from Althea Health, personal fees from Dova Pharmaceuticasl, outside the submitted work. Dr. GS reports personal fees from Medtronic Vascular, outside the submitted work. The authors have no other conflicts of interest to declare.

Ethical Statement: The authors are accountable for all aspects of the work in ensuring that questions related to the accuracy or integrity of any part of the work are appropriately investigated and resolved.

Open Access Statement: This is an Open Access article distributed in accordance with the Creative Commons Attribution-NonCommercial-NoDerivs 4.0 International License (CC BY-NC-ND 4.0), which permits the noncommercial replication and distribution of the article with the strict proviso that no changes or edits are made and the original work is properly cited (including links to both the formal publication through the relevant DOI and the license). See: https://creativecommons.org/licenses/by-nc$\mathrm{nd} / 4.0 \%$.

\section{References}

1. Burke RM, Rayan SS, Kasirajan K, et al. Unusual case of right-sided May-Thurner syndrome and review of its management. Vascular 2006;14:47-50.

2. Fretz V, Binkert CA. Compression of the inferior vena cava by the right iliac artery: a rare variant of May-Thurner syndrome. Cardiovasc Intervent Radiol 2010;33:1060-3.

3. Virchows Archiv. A, Pathological anatomy and histology. - NLM Catalog - NCBI [Internet]. [cited 2020 Jan 11]. Available online: https://www.ncbi.nlm.nih.gov/ nlmcatalog?term $=0340-1227 \% 5$ BISSN \% 5D

4. Mcmurrich JP. The occurrence of congenital adhesions in the common iliac veins, and their relation to thrombosis of the femoral and iliac veins. Am J Med Sci 1908;135:342-5.

5. Ehrich WE, Krumbhaar EB. A frequent obstructive anomaly of the mouth of the left common iliac vein. Am Heart J 1943;26:737-50.
6. May R, Thurner J. The cause of the predominantly sinistral occurrence of thrombosis of the pelvic veins. Angiology 1957;8:419-27.

7. Cockett FB, Thomas ML. The iliac compression syndrome. Br J Surg 1965;52:816-21.

8. Brazeau NF, Harvey HB, Pinto EG, et al. May-Thurner syndrome: diagnosis and management. Vasa 2013;42:96105.

9. Kalu S, Shah P, Natarajan A, et al. May-thurner syndrome: a case report and review of the literature. Case Rep Vasc Med 2013;2013:740182.

10. Eberhardt RT, Raffetto JD. Chronic venous insufficiency. Circulation 2005;111:2398-409.

11. Delis KT, Bountouroglou D, Mansfield AO. Venous claudication in iliofemoral thrombosis: long-term effects on venous hemodynamics, clinical status, and quality of life. Ann Surg 2004;239:118-26.

12. Oguzkurt L, Ozkan U, Ulusan S, et al. Compression of the left common iliac vein in asymptomatic subjects and patients with left iliofemoral deep vein thrombosis. J Vasc Interv Radiol 2008;19:366-70; quiz 371.

13. Zander KD, Staat B, Galan H. May-Thurner Syndrome resulting in acute iliofemoral deep vein thrombosis in the postpartum period. Obstet Gynecol 2008;111:565-9.

14. Murphy EH, Davis CM, Journeycake JM, et al. Symptomatic ileofemoral DVT after onset of oral contraceptive use in women with previously undiagnosed May-Thurner Syndrome. J Vasc Surg 2009;49:697-703.

15. Chung JW, Yoon CJ, Jung SI, et al. Acute iliofemoral deep vein thrombosis: evaluation of underlying anatomic abnormalities by spiral CT venography. J Vasc Interv Radiol 2004;15:249-56.

16. Wolpert LM, Rahmani O, Stein B, et al. Magnetic resonance venography in the diagnosis and management of May-Thurner syndrome. Vasc Endovascular Surg 2002;36:51-7.

17. Gurel K, Gurel S, Karavas E, et al. Direct contrastenhanced MR venography in the diagnosis of MayThurner syndrome. Eur J Radiol 2011;80:533-6.

18. Jeon UB, Chung JW, Jae HJ, et al. May-Thurner syndrome complicated by acute iliofemoral vein thrombosis: helical CT venography for evaluation of longterm stent patency and changes in the iliac vein. AJR Am J Roentgenol 2010;195:751-7.

19. Butros SR, Liu R, Oliveira GR, et al. Venous compression syndromes: clinical features, imaging findings and management. Br J Radiol 2013;86:20130284.

20. Markl M, Frydrychowicz A, Kozerke S, et al. 4D flow 
MRI. J Magn Reson Imaging 2012;36:1015-36.

21. Sträter A, Huber A, Rudolph J, et al. 4D-Flow MRI: Technique and Applications. RöFo - Fortschritte Auf Dem Geb Röntgenstrahlen Bildgeb Verfahr 2018;190:1025-35.

22. Oliveira IS, Hedgire SS, Li W, et al. Blood pool contrast agents for venous magnetic resonance imaging. Cardiovasc Diagn Ther 2016;6:508-18.

23. Brinegar KN. Iliac vein compression syndrome: Clinical, imaging and pathologic findings. World J Radiol 2015;7:375.

24. Behzadi AH, Khilnani NM, Zhang W, et al. Pelvic cardiovascular magnetic resonance venography: venous changes with patient position and hydration status. J Cardiovasc Magn Reson 2019;21:3.

25. Zucker EJ, Ganguli S, Ghoshhajra BB, et al. Imaging of venous compression syndromes. Cardiovasc Diagn Ther 2016;6:519-32.

26. Ferris EJ, Lim WN, Smith PL, et al. May-Thurner syndrome. Radiology. 1983;147:29-31.

27. Vedantham S, Goldhaber SZ, Julian JA, et al. Pharmacomechanical Catheter-Directed Thrombolysis for Deep-Vein Thrombosis. N Engl J Med 2017;377:2240-52.

28. Kaltenmeier CT, Erben Y, Indes J, et al. Systematic review of May-Thurner syndrome with emphasis on gender differences. J Vasc Surg Venous Lymphat Disord 2018;6:399-407.e4.

29. Birn J, Vedantham S. May-Thurner syndrome and other obstructive iliac vein lesions: meaning, myth, and mystery. Vasc Med 2015;20:74-83.

30. Meissner MH, Gloviczki P, Comerota AJ, et al. Early thrombus removal strategies for acute deep venous thrombosis: Clinical Practice Guidelines of the Society for
Vascular Surgery and the American Venous Forum. J Vasc Surg 2012;55:1449-62.

31. Shi WY, Gu JP, Liu CJ, et al. Endovascular treatment for iliac vein compression syndrome with or without lower extremity deep vein thrombosis: A retrospective study on mid-term in-stent patency from a single center. Eur J Radiol 2016;85:7-14.

32. Neglén P, Hollis KC, Olivier J, et al. Stenting of the venous outflow in chronic venous disease: Long-term stent-related outcome, clinical, and hemodynamic result. J Vasc Surg 2007;46:979-90.

33. Bajwa R, Bergin D, O'Sullivan GJ. Aiming for the Bottom Corner: How to Score a Field Goal When Landing Venous Stents in May-Thurner Syndrome. J Vasc Interv Radiol 2019;30:1555-61.

34. Lichtenberg M, Breuckmann F, Friedrich Stahlhoff W, et al. Placement of closed-cell designed venous stents in a mixed cohort of patients with chronic venous outflow obstructions - short-term safety, patency, and clinical outcomes. Vasa 2018;47:475-81.

35. Lichtenberg MKW, de Graaf R, Stahlhoff WF, et al. Venovo venous stent in the treatment of non-thrombotic or post-thrombotic iliac vein lesions - short-term results from the Arnsberg venous registry. Vasa 2019;48:175-80.

36. J Padrnos L, Garcia D. May-Thurner syndrome and thrombosis: A systematic review of antithrombotic use after endovascular stent placement. Res Pract Thromb Haemost 2018;3:70-8.

37. Yin M, Huang X, Cui C, et al. The effect of stent placement for May-Thurner syndrome combined with symptomatic superficial venous reflux disease. J Vasc Surg Venous Lymphat Disord 2015;3:168-72.
Cite this article as: Poyyamoli S, Mehta P, Cherian M, Anand RR, Patil SB, Kalva S, Salazar G. May-Thurner syndrome. Cardiovasc Diagn Ther 2021;11(5):1104-1111. doi: 10.21037/ cdt.2020.03.07 